\title{
Dolosigranulum pigrum: Predicting Severity of Infection
}

\author{
John Sherret ${ }^{1}$, Bhavesh Gajjar ${ }^{1}$, Lamis Ibrahim ${ }^{2}$, Ahmed Mohamed Ahmed ${ }^{2}$, Utsab R. Panta ${ }^{1}$ \\ 1. Internal Medicine, East Tennessee State University Quillen College of Medicine, Johnson City, USA 2. Infectious \\ Disease, East Tennessee State University Quillen College of Medicine, Johnson City, USA
}

Corresponding author: John Sherret, sherret@etsu.edu

\begin{abstract}
In this report, we describe a case of a 61-year-old male patient who had the bacterium Dolosigranulum pigrum growing in a blood culture. It was susceptible to ampicillin, ceftriaxone, levofloxacin, and vancomycin but was intermediately resistant to erythromycin. The patient did not have a negative outcome as a consequence of this bacterium, which retrospectively could have been predicted based on the epidemiological data within the patient's profile.
\end{abstract}

Categories: Internal Medicine, Infectious Disease, Epidemiology/Public Health

Keywords: dolosigranulum pigrum, antibiotic susceptibility, opportunistic infection, immunocompromised

\section{Introduction}

Dolosigranulum pigrum (D. pigrum) was first reported in 1993 when 16S RNA gene sequencing revealed previously uncharacterized gram-positive catalase-negative bacteria [1]. Since discovery the reporting of this bacterium has been increasing. While this increased incidence could be due to increased usage of RNA gene sequencing in clinical laboratories, it also could mark the emergence of an opportunistic infection particularly in immunocompromised patients [2]. Otherwise, it has been suggested that $D$. pigrum remains a commensal organism [2-5].

D. pigrum can cause eye infections, sepsis, nosocomial pneumonia, and ventilator-associated pneumonia $[2,6,7]$. It has been suspected in synovitis and cholecystitis and has also been isolated from gastric contents, blood and urine cultures, the nasopharyngeal regions, and even in the spinal cord $[6,8,9]$. D. pigrum has been reported in patients as young as two months and as old as 85 years [6]. Currently, 27 strains have been identified [10]. All strains are susceptible to beta-lactam antibiotics, and approximately half of the strains are known to be resistant to erythromycin $[2,6]$. In this report, we present a sighting of this bacterium, its antibiotic susceptibilities, and the risk factors that predict illness.

Received 07/25/2020

Review began 08/02/2020 Review ended 08/08/2020 Published 08/15/2020

\section{Copyright 2020}

Sherret et al. This is an open access article distributed under the terms of the Creative Commons Attribution License CC-BY 4.0., which permits unrestricted use, distribution, and reproduction in any medium, provided the original author and source are credited.

\section{Case Presentation}

Our patient was a 61-year-old male who presented to the emergency room for shortness of breath. He was hypoxic, requiring two liters of oxygen but his other vital signs were within a normal range. He also complained of bilateral leg swelling but denied fever, chills, cough, wheezing, palpitations, and dizziness. He was a former smoker and denied alcohol or illicit drug use. The patient had recently completed six weeks of cefepime and metronidazole for right foot osteomyelitis. He had a past medical history of heart failure with reduced ejection fraction, end-stage renal disease on hemodialysis, type II diabetes, asthma, stroke, and coronary artery disease with prior myocardial infarction and percutaneous intervention. Chest and foot X-rays did not show any concerns for infection.

He was treated for an acute exacerbation of congestive heart failure. He was given a one-time dose of cefepime in the emergency department and was continued on hemodialysis. Two days into admission, the blood culture revealed non-specific gram-positive organisms for which vancomycin was started. Five days later, the microbe was identified as D. pigrum and repeat blood cultures obtained six days after admission were negative. The susceptibilities showed $D$. pigrum to be pan-sensitive except for erythromycin which demonstrated intermediate resistance (Table 1 ). 


\section{Cureus}

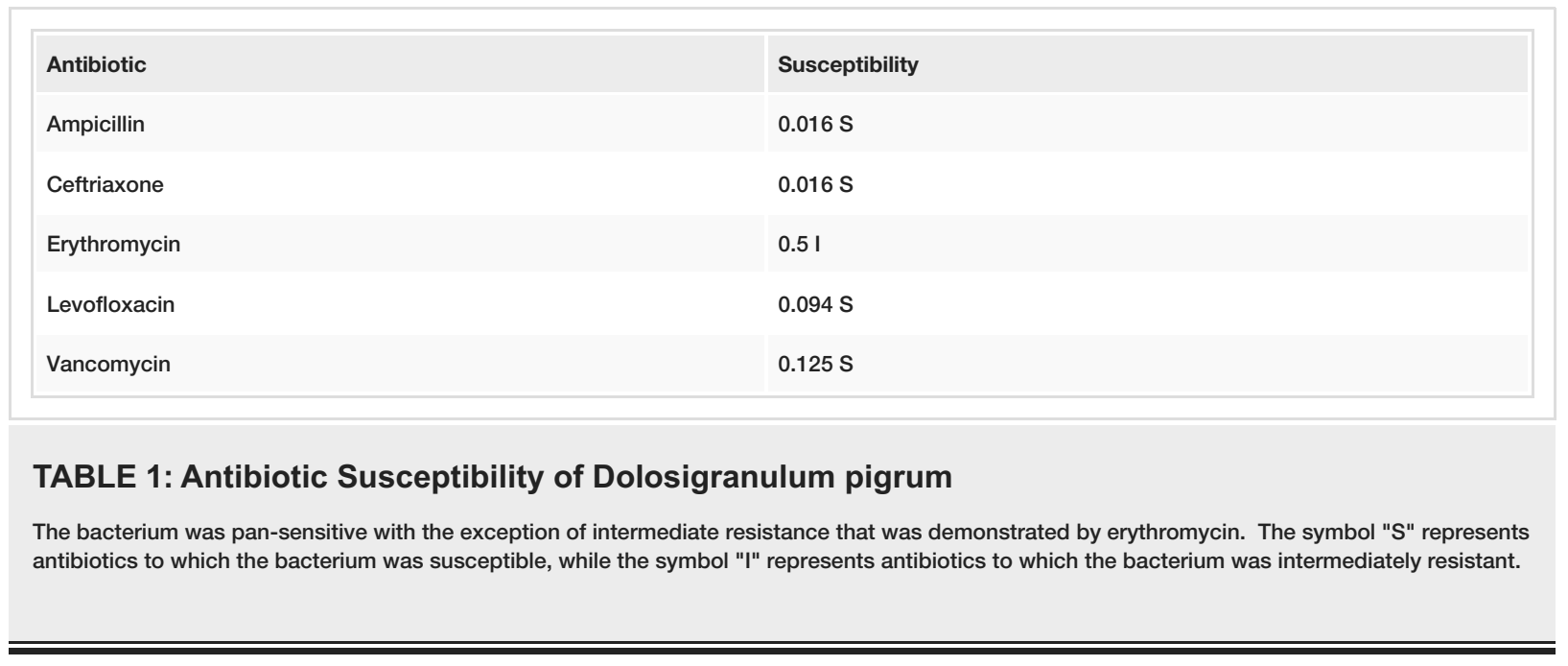

\section{Discussion}

The understanding of the general pathology and epidemiology of $D$. pigrum has been growing over time. Many studies have documented asymptomatic $D$. pigrum residency in the upper respiratory tracts [11-13]. However, several case reports to date have documented a variety of infections. One report mentions a 64year-old male with rheumatoid arthritis who was taking chronic prednisone and methotrexate to control synovitis. His left arm became very tender. Blood cultures were drawn, antibiotics were started, and then an arthrocentesis was performed. While the blood cultures were positive for D. pigrum, the synovial fluid was sterile but had numerous amounts of leukocytes and followed an infectious pattern [8]. Another report is of a 73-year-old male with a medical history of chronic obstructive pulmonary disease due to heavy smoking, thrombocythemia treated with hydroxycarbamide, and childhood pulmonary tuberculosis who presented for acute respiratory insufficiency due to pulmonary embolism. Thirteen days into admission, the patient developed fever and blood cultures were drawn revealing $D$. pigrum. Two days later, he developed septic shock requiring emergent intubation and bronchoalveolar lavage which was positive for Staphylococcus aureus and D. pigrum [2]. In a third report, 25 patients with cystic fibrosis who were hospitalized provided sputum samples which detected D. pigrum and others [14]. Given the current knowledge that pulmonary infections in cystic fibrosis are polymicrobial, such detections are significant [14-18]. In a fourth report, a 51year-old male with no past medical history was admitted for severe aneurysmal subarachnoid hemorrhage with respiratory distress and was subsequently intubated. On ventilator day 10, he developed signs of pneumonia and cultures of bronchial secretions ultimately revealed D. pigrum [7]. In a fifth report, D. pigrum was suspected to be the cause of an acute cholecystitis in a 76-year-old male with gallstones and no other past medical history. The blood cultures were positive for D. pigrum [9]. In a sixth report, the Centers for Disease Control and Prevention (CDC) reported on 27 isolates they had received. Twelve had come from blood cultures, nearly half of which had sepsis. Six were from the eye, four were from the nasopharynx, one was from the sinus, one was from sputum, and one was from the stomach suggesting an upper respiratory habitat for the bacterium [6]. Many of these patients were either less than 2 years of age or older than 65 years of age. In a seventh report, a 71-year-old female, an 85-year-old male, and a 78-year-old female all had keratitis with corneal specimens positive for D. pigrum [4]. This further suggests that $D$. pigrum is an emerging pathogen that particularly targets the immunocompromised and the elderly.

In comparison to prior reports, our patient followed patterns that predicted a favorable outcome [2-9,11-15]. He was a 61-year-old male with no chronic immunosuppressive conditions or medications apart from the end-stage renal disease on dialysis due to type II diabetes. He was less than 65 years of age. Therefore, he was unlikely to experience septic shock or severe respiratory distress from this organism. While he presented with shortness of breath, it was not severe as the pulse saturation was $99 \%$ on two liters of oxygen. Only one of the blood cultures initially revealed a gram-positive organism which was treated with vancomycin. A transthoracic echocardiogram was negative for endocarditis. On day 6, the initial blood culture identified the organism as D. pigrum. Two repeat peripheral cultures and a culture obtained from the dialysis ash catheter were negative. The patient was afebrile and never exhibited a leukocytosis. Given that the patient was immunocompetent and younger than 65 years, he was unlikely to experience a serious infection. Future cases would be wise to consider these criteria in D. pigrum infections.

The bacterium $D$. pigrum is mostly pan-sensitive to conventional antibiotics. Our specimen was sensitive to beta-lactams, cephalosporin, fluoroquinolones, and vancomycin. However, it was intermediately resistant to erythromycin, which largely matched the CDC data $[4,6-9]$. The CDC data showed that $52 \%$ of their isolates were resistant to erythromycin, with most of those resistant samples appearing in corneal collections [6]. Despite our patient being on six weeks of cefepime and metronidazole for right foot osteomyelitis, the $D$. pigrum was pan-sensitive. These observations suggest that $D$. pigrum does not contain widespread antibiotic resistance. 


\section{Conclusions}

D. pigrum is a potential emerging opportunistic infection that favors those $>65$ and $<2$ years of age, the immunocompromised, and those with baseline pulmonary diseases, such as cystic fibrosis and severe chronic obstructive pulmonary disease. $D$. pigrum appears to be very susceptible to current antibiotics. It can be challenging to identify in the laboratory setting which can cause delays in obtaining results. Further research is required to obtain a more accurate incidence and rapid diagnosis of the bacterium.

\section{Additional Information \\ Disclosures}

Human subjects: Consent was obtained by all participants in this study. Conflicts of interest: In compliance with the ICMJE uniform disclosure form, all authors declare the following: Payment/services info: All authors have declared that no financial support was received from any organization for the submitted work. Financial relationships: All authors have declared that they have no financial relationships at present or within the previous three years with any organizations that might have an interest in the submitted work. Other relationships: All authors have declared that there are no other relationships or activities that could appear to have influenced the submitted work.

\section{References}

1. Aguirre M, Morrison D, Cookson BD, Gay FW, Collins MD: Phenotypic and phylogenetic characterization of some gemella-like organisms from human infections: description of Dolosigranulum pigrum gen. nov., sp. nov. J Appl Bacteriol. 1993, 75:608-612. 10.1111/j.1365-2672.1993.tb01602.x

2. Lécuyer H, Audibert J, Bobigny A, et al.: Dolosigranulum pigrum causing nosocomial pneumonia and septicemia. J Clin Microbiol. 2007, 45:3474-3475. 10.1128/JCM.01373-07

3. Ortiz Moyano R, Raya Tonetti F, Tomokiyo M, et al.: The ability of respiratory commensal bacteria to beneficially modulate the lung innate immune response is a strain dependent characteristic. Microorganisms. 2020, 8:727. 10.3390/microorganisms8050727

4. Sampo M, Ghazouani O, Cadiou D, Trichet E, Hoffart L, Drancourt M: Dolosigranulum pigrum keratitis: a three-case series. BMC Ophthalmol. 2013, 13:31. 10.1186/1471-2415-13-31

5. Song C, Chorath J, Pak Y, Redjal N: Use of dipstick assay and rapid PCR-DNA analysis of nasal secretions for diagnosis of bacterial sinusitis in children with chronic cough. Allergy Rhinol (Providence). 2019, 10:1-8. $10.1177 / 2152656718821281$

6. LaClaire LL, Facklam RR: Antimicrobial susceptibility and clinical sources of Dolosigranulum pigrum cultures. Antimicrob Agents Chemother. 2000, 44:2001-2003. 10.1128/aac.44.7.2001-2003.2000

7. Hoedemaekers A, Schülin T, Tonk B, Melchers WJ, Sturm PD: Ventilator-associated pneumonia caused by Dolosigranulum pigrum. J Clin Microbiol. 2006, 44:3461-3462. 10.1128/JCM.01050-06

8. Hall GS, Gordon S, Schroeder S, Smith K, Anthony K, Procop GW: Case of synovitis potentially caused by Dolosigranulum pigrum. J Clin Microbiol. 2001, 39:1202-1203. 10.1128/JCM.39.3.1202-1203.2001

9. Lin JC, Hou SJ, Huang LU, Sun JR, Chang WK, Lu JJ: Acute cholecystitis accompanied by acute pancreatitis potentially caused by Dolosigranulum pigrum. J Clin Microbiol. 2006, 44:2298-2299. 10.1128/JCM.02520-05

10. LaClaire LL, Facklam RR: Comparison of three commercial rapid identification systems for the unusual gram-positive cocci Dolosigranulum pigrum, Ignavigranum ruoffiae, and Facklamia species. J Clin Microbiol. 2000, 38:2037-2042. 10.1128/JCM.38.6.2037-2042.2000

11. Bogaert D, Keijser B, Huse S, et al.: Variability and diversity of nasopharyngeal microbiota in children: a metagenomic analysis. PLoS One. 2011, 6:e17035. 10.1371/journal.pone.0017035

12. Camarinha-Silva A, Wos-Oxley ML, Jáuregui R, Becker K, Pieper DH: Validating T-RFLP as a sensitive and high-throughput approach to assess bacterial diversity patterns in human anterior nares. FEMS Microbiol Ecol. 2012, 79:98-108. 10.1111/j.1574-6941.2011.01197.x

13. Wos-Oxley ML, Plumeier I, von Eiff C, et al.: A poke into the diversity and associations within human anterior nare microbial communities. ISME J. 2010, 4:839-851. 10.1038/ismej.2010.15

14. Bittar F, Richet H, Dubus JC, et al.: Molecular detection of multiple emerging pathogens in sputa from cystic fibrosis patients. PLoS One. 2008, 3:e2908. 10.1371/journal.pone.0002908

15. Rogers GB, Hart CA, Mason JR, Hughes M, Walshaw MJ, Bruce KD: Bacterial diversity in cases of lung infection in cystic fibrosis patients: 16S ribosomal DNA (rDNA) length heterogeneity PCR and 16S rDNA terminal restriction fragment length polymorphism profiling. J Clin Microbiol. 2003, 41:3548-3558. 10.1128/jcm.41.8.3548-3558.2003

16. Rogers GB, Carroll MP, Serisier DJ, Hockey PM, Jones G, Bruce KD: Characterization of bacterial community diversity in cystic fibrosis lung infections by use of $16 \mathrm{~S}$ ribosomal DNA terminal restriction fragment length polymorphism profiling. J Clin Microbiol. 2004, 42:5176-5183. 10.1128/JCM.42.11.5176-5183.2004

17. Kolak M, Karpati F, Monstein HJ, Jonasson J: Molecular typing of the bacterial flora in sputum of cystic fibrosis patients. Int J Med Microbiol. 2003, 293:309-317. 10.1078/1438-4221-00265

18. van Belkum A, Renders NH, Smith S, Overbeek SE, Verbrugh HA: Comparison of conventional and molecular methods for the detection of bacterial pathogens in sputum samples from cystic fibrosis patients. FEMS Immunol Med Microbiol. 2000, 27:51-57. 10.1111/j.1574-695X.2000.tb01411.x 